\title{
Construction d'un barrage mobile sur une voie navigable à fort trafic
}

\section{Construction of a gated weir on a waterway carrying heavy traffic}

\author{
R. Lefoulon
}

Inspecteur Général Honoraire d'Électricité de France

\begin{abstract}
L'aménagement du Rhin entre Bâle et Strasbourg qui se poursuivait par la construction des biefs successifs du grand canal d'Alsace, parallèlement au fleuve, fut modifié en octobre 1956 par les accords franco. allemands. A partir de cette date les quatre biefs restant à réaliser devaient faire l'objet d'un retour au Rhin sur au moins la moitié de la longueur du bief. Autrement dit chaque bief devait comporter un barrage de dérivation.

Ainsi pour poursuivre, sans discontinuité, l'aménagement du Rhin nous étions amenés à concevoir fin 1956, dans le plus court délai, le barrage du bief de Marckolsheim pour le soumettre à la Commission Internationale du Rhin. Le projet de l'ouvrage en lui même ne présentait pas de difficultés mais sa construction dans le lit du Rhin posait deux problèmes : d'une part, les
\end{abstract}

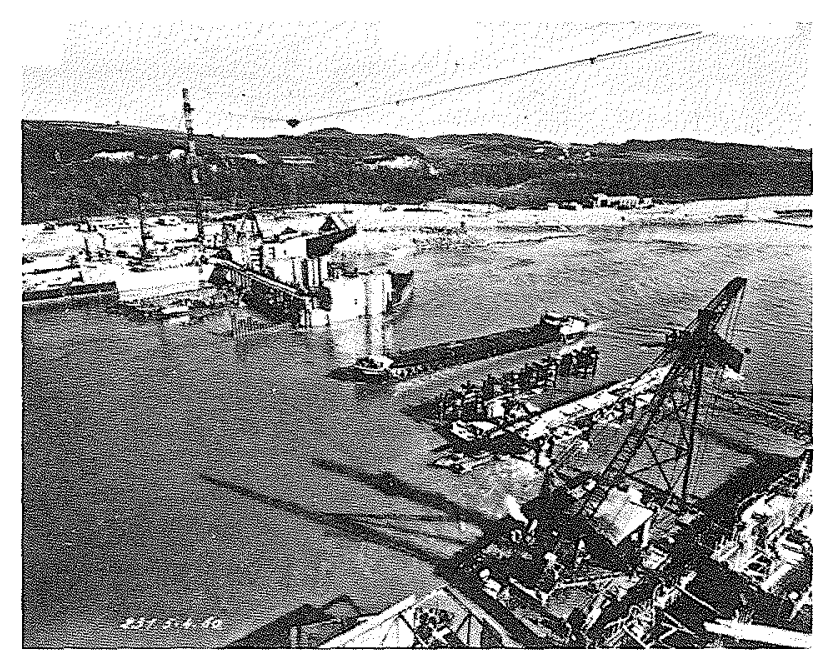

Vue générale du chantier $2^{\circledR}$ phase (avril 1960). fondations, d'autre part, le maintien d'une navigation à fort trafic à travers le chantier pendant les travaux.

Pour l'exécution des fondations on limita le nombre des passes à 5 de $30 \mathrm{~m}$. Les quatre piles dans le fleuve furent construites par fonçage de caisson à air comprimé s'enfonçant de $10 \mathrm{~m}$ dans les alluvions. Cette fondation fut complètée par une coupure jusqu'à la Marne par une injection des alluvions sur $10 \mathrm{~m}$ de largeur.

Pour assurer la navigation sans interruption, les phases de travaux furent étudiées sur un modèle réduit de $6 \mathrm{~km}$ de Rhin à l'échelle 1/66 aux Laboratoires de Chatou.

Il nous fallait faire face à une navigation annuelle de 7 millions de tonnes c'est-à-dire au passage journalier de 80 à 125 bateaux comprenant des automoteurs de $2000 \mathrm{ch}$. et des convois tractés par des remorqueurs

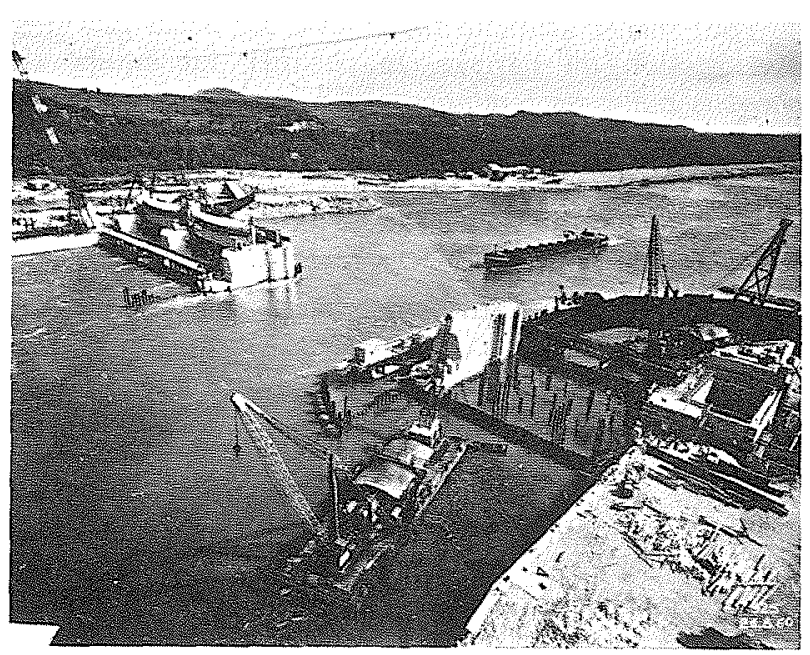

Vue générale du chantier, $3^{\mathbb{e}}$ phase (août 1960).

LA HOUILLE BLANCHE/N ${ }^{\circ} 2 / 3-1981$ 


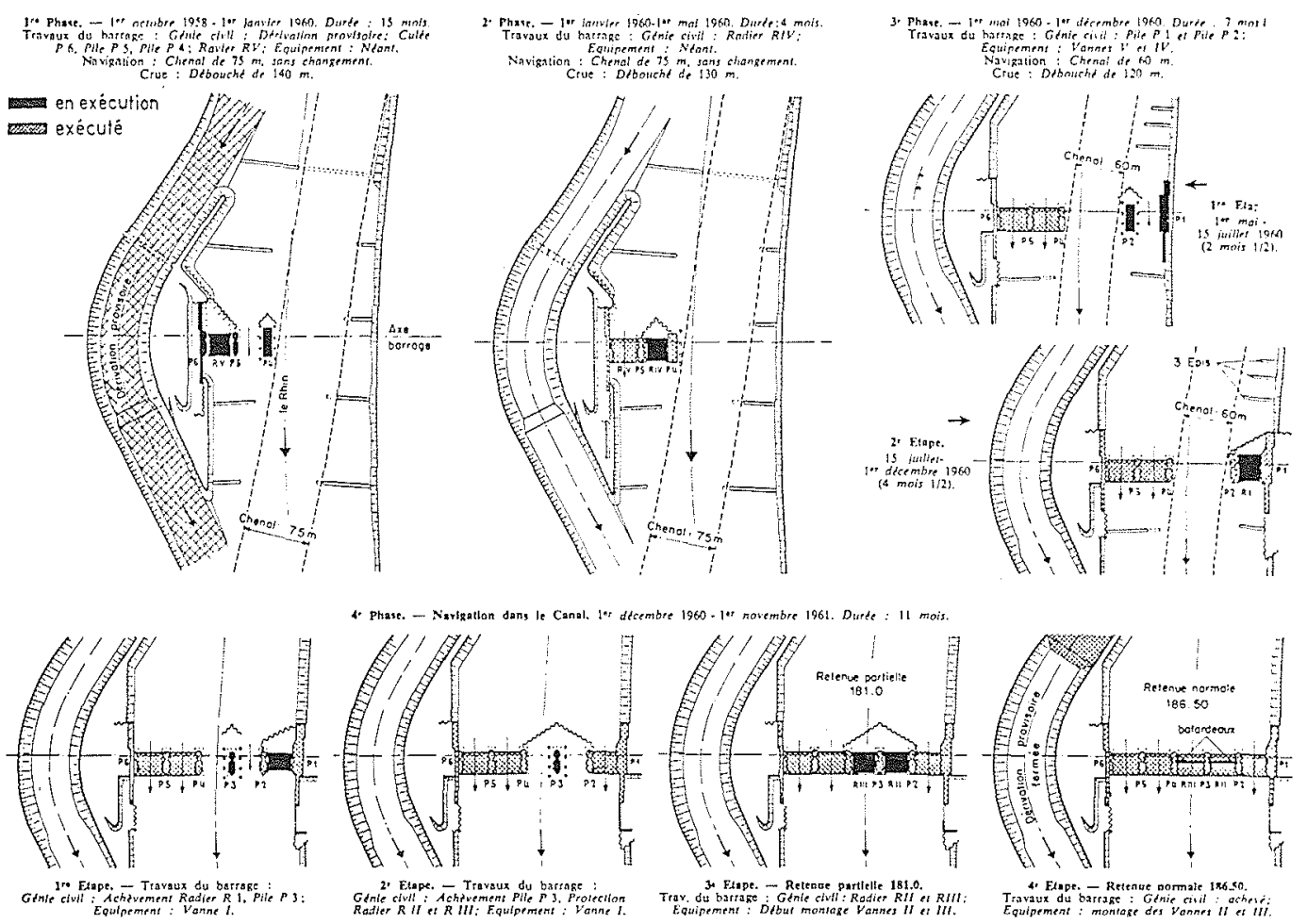

Figure 1 - Aménagement de Marckolsheim. Programme des Travaux.

de 3000 ch. et ceci entre le débit de $540 \mathrm{~m}^{3} / \mathrm{sec}$. (plus basses eaux navigables) et de $2800 \mathrm{~m}^{3} / \mathrm{sec}$. (plus hautes eaux navigables) - Par ailleurs il fallait assurer le passage de la crue décennale de $3500 \mathrm{~m}^{3} / \mathrm{sec}$. -

En premier lieu, le modèle reduit permit de définir les dimensions et les côtes d'une dérivation provisoire ayant la double fonction de réduire le débit de $20 \%$ dans le chenal navigable pour le débit maximum de $2800 \mathrm{~m}^{3} / \mathrm{sec}$. et de maintenir la presque totalité du débit dans le chenal pendant les basses eaux - Puis le modèle fut utilisé en permanence pour définir les quatre phases de travaux représentées sur la figure 1 qui maintenaient un chenal navigable de 60 mètres approuvé par la Commission Centrale du Rhin.

Pour mener à bonne fin et dans les délais prévus la construction du barrage, le chantier fut équipé de moyens puissants : remorqueur de $460 \mathrm{ch}$., Blondin de $300 \mathrm{~m}$ avec des pylones de $60 \mathrm{~m}$ de hauteur. Des pontons équipés de derrick de $90 \mathrm{t} / \mathrm{m}$ et $60 \mathrm{t} / \mathrm{m}$ et de treuils de manœuvre puissants, une drague, sans oublier les caissons à air comprimé.

En présence des difficultés et des moyens mis en œuvre pour la construction du barrage de Marckolsheim, on comprendra aisément que lorsqu'il s'est agi de projeter, en 1958, le barrage du bief de Rhinau faisant suite à celui de Marckolsheim nous ayons recherché une autre solution. C'est cette solution qui fait l'objet de notre communication d'aujourd'hui.

La conception originale du barrage mobile de Rhinau consiste à construire le barrage mobile dans sa totalité sur une des rives du fleuve, en dehors du lit, et de pro- longer ce barrage par une digue de coupure du fleuve c'est-à-dire par un barrage fixe en alluvions et enrochement.

Pendant une première période, la plus longue (environ 3 ans), on exécute sur l'une ou l'autre rive les ou-
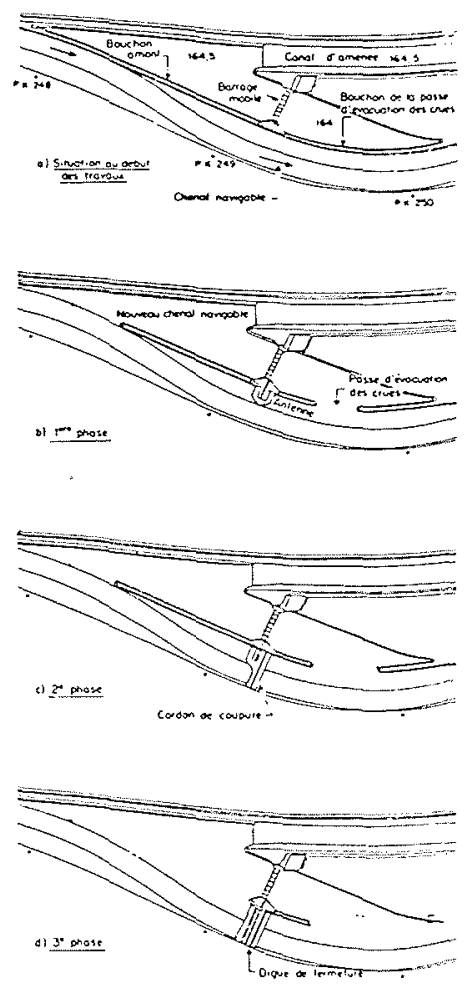

Figure 2. - Phases de mise en service de l'aménagement de Rhinau 
vrages de l'aménagement du bief non seulement le barrage mobile mais également le canal de dérivation, les écluses et l'usine hydroélectrique. Pendant toute cette période la navigation reste dans le fleuve. Les fondations et la superstructure du barrage mobile sont réalisées avec des moyens et engins classiques : Rideaux de palplanches - rabattement, grues pour mise en place du béton - Pour réaliser l'ouvrage le plus économique rien ne s'oppose à augmenter le nombre des passes à 7 de $20 \mathrm{~m}$.

Le chantier du barrage est protégé des crues jusqu'à $3500 \mathrm{~m}^{3} / \mathrm{sec}$. par des petites digues surélevant les berges à l'amont et à l'aval du chantier. Les cotes du seuil amont du barrage et de plafond du canal d'amenée sont fixées pour avoir un tirant d'eau de $2 \mathrm{~m}$ au moment de la mise en eau dans l'hypothèse du débit minimum navigable de $540 \mathrm{~m}^{3} / \mathrm{sec}$. Ces dispositions sont étudiées ainsi que les phases de la $2^{\mathrm{e}}$ période de construction sur un modèle réduit au $1 / 66^{\mathrm{e}}$ de $6 \mathrm{~km}$ de Rhin au laboratoire de Chatou.

Les phases de cette $2^{\mathrm{e}}$ période sont représentées sur la figure 2 ; pendant la $1{ }^{\text {ère }}$ phase on procède :

a) à l'enlèvement des bouchons à l'amont et à l'aval du barrage mobile. Celui-ci et le canal d'amenée sont alors en communication avec le Rhin. La navigation est toujours maintenue dans le fleuve ;

b) avec les déblais, on réalise l'amorce de la digue par une antenne de $60 \mathrm{~m}$ laissant libre sur $140 \mathrm{~m}$ le chenal de navigation.

La durée relativement faible ( 40 jours environ) de cette phase permet de la réaliser à une période ou le débit est inférieur à $2000 \mathrm{~m}^{3} / \mathrm{sec}$. A la fin de cette phase, barrage mobile, canal de dérivation et écluses sont prêts à la mise en service et au passage de la navigation.

La seconde phase est la plus délicate, on y réalise la coupure du fleuve le plus rapidement possible. De fait elle fut exécutée en 7 jours par la mise en place d'un tapis d'enrochement puis d'un cordon trapézoidale de $1200 \mathrm{~m}^{3}$ au moyen de chalands à clapet et de camions de terrassement. Sans problème, la navigation emprunte le canal de navigation dès le début de cette phase et $y$ sera maintenue.

La troisième phase est celle de la construction de la digue de fermeture. Le niveau dans le canal d'amenée s'élève progressivement jusqu'à la cote de retenue normale.

La construction du barrage de Rhinau n'a donné lieu à aucune difficulté pour la navigation pendant la durée des travaux. A cet avantage très important s'ajoute une économie dans le coût de l'ouvrage par rapport au barrage de Marckolsheim de 20 millions de francs lourds de 1962; économie résultant de l'exécution du génie civil en dehors du lit du fleuve et de l'allégement de la ventellerie de $1800 \mathrm{~T}$ à $1000 \mathrm{~T}$.

La conception de Rhinau a été adoptée pour la construction des quatre barrages sur le Rhin à l'aval de Rhinau. Elle peut être recommandée pour la construction de barrage mobile sur les voies navigables à fort trafic. 